\title{
De Frankfurt a Rio Cuarto: cuestiones para el diálogo analógico de la Teoría Crítica y la Filosofía de la Liberación*
}

From Frankfurt to Rio Cuarto: Issues for the analogue dialogue of the Critical Theory and the Philosophy of Liberation

De Frankfurt ao Rio Cuarto: questões para o diálogo analógico da Teória Crítica e a Filosofia da Libertação

Fecha de entrega: 29 de junio de 2016

Fecha de evaluación: 5 de julio de 2016

Fecha de aprobación: 30 de julio de 2016

Abdiel Rodríguez Reyes ${ }^{* *}$

\section{Resumen}

Este artículo como objetivos plantea el tema de la necesidad de diálogo analógico de la Teoría Crítica con la Filosofía de la Liberación, fundamentalmente entre las nuevas generaciones, para aunar esfuerzos en la crítica sistemática al capitalismo y la modernidad. Ver algunas relaciones que se dieron en el siglo XX, entre los pensadores de la Escuela de Frankfurt y Latinoamérica, que las nuevas generaciones tendrían

* Este artículo forma parte de la actividad investigadora del autor en el Centro de Investigación de la Facultad de Humanidades de la Universidad de Panamá. DOI: http://dx.doi.org/10.15332/ s0120-8462.2016.0115.01

* Investigador en la Universidad de Panamá. Candidato a doctor en Filosofía por la Universidad del País Vasco. Correo electrónico: abdiel.rodriguez@up.ac.pa 
que retomar. Y, por último, ver las condiciones de posibilidad de dicho diálogo analógico.

Palabras clave: Filosofía, pensamiento, comunicación intercultural, capitalismo, crítica.

\section{Abstract}

This paper has as objectives, propose the issue of the need for analog dialogue between Critical Theory with the Philosophy of Liberation, fundamentally among the new generations, towards a concerted effort a systematic criticism of capitalism and modernity. View some relationships that had place in XX century, between the thinkers of the Frankfurt School and Latin America that new generations should retake. And finally, see the conditions of possibility of such analog dialogue.

Keywords: Philosophy, thinking, intercultural communication, capitalism, criticism.

\section{Resumo}

Este artigo como objetivo apresenta o tema da necessidade de dialogo analógico da Teoria Crítica com a Filosofia da Libertação, fundamentalmente entres as novas gerações, para unir esforços na crítica sistemática ao capitalismo e a modernidade. Observar algumas relações que aconteceram no século XX entre os pensadores da Escola de Frankfurt e Latino-américa que as novas gerações teriam que retomar. Por último, ver as condições de possibilidade de este diálogo análogo.

Palavras-chave: Filosofia, pensamento, comunicação intercultural, capitalismo, critica. 


\section{Introducción}

Vivimos en tiempos de ebullición, en donde todo lo sólido se desvanece en el aire (Marx y Engels, 1976, p. 27) ${ }^{1}$. Es el momento de la inmediatez, de la big data y el smartphone. Ante esta abrumadora realidad, como una planta en el proceso de fotosíntesis, buscamos los pocos rayos de luz que quedan. En nuestro caso, en la tradición del pensamiento crítico mundial; en particular, en la Teoría Crítica y la Filosofía de la Liberación.

Antes de hablar sobre las cuestiones para un virtual diálogo analógico entre la Teoría Crítica ${ }^{2}$ que se originó en Frankfurt y la Filosofía de la Liberación ${ }^{3}$ que se originó en Argentina, y se reafirmó en Rio Cuarto ${ }^{4}$, ambas con impacto mundial ${ }^{5}$, quiero hacer un breve recuento de cómo algunos pensadores de la Escuela de Frankfurt, y de lo que potencialmente sería la Teoría Crítica, mantuvieron algún vínculo, alguna relación con Latinoamérica, cuestión que se dio de diversas formas. Ahora bien, hacer un recuento total de estas relaciones es un esfuerzo que rebasa los límites y fines de este artículo. Por lo cual, solo nos centraremos en algunos casos, para tener una idea al respecto.

Estas relaciones no se dieron de forma homogénea, tampoco tuvieron el mismo impacto en todos los países. Empero, si es palpable hasta nuestros días, una fuerte

1 Y, el interesantisimo libro de Berman del año 1998: Todo lo sólido se desvanece en el aire. La experiencia de la modernidad.

2 "La Teoría Crítica la desarrollaron los miembros del círculo que se formó en torno a Horkheimer, en un intento de dar razón de los desengaños políticos que representaron el fracaso de la revolución en occidente, la evolución de la Rusia estalinista y la victoria del fascismo en Alemania; la Teoria Crítica se propuso explicar el fracaso de los pronósticos marxistas, sin romper, empero, con las intenciones del marxismo" (Habermas, 1993, p. 146).

3 Tomamos a la Filosofía de la Liberación como virtual interlocutora, porque es en lo que estamos trabajando, además que consideramos que es uno de los esfuerzos más originales, creativos y críticos del filosofar en Latinoamérica, además que con Beorlegui (2010, p. 661) (él lo plantea en plural, que es válido, ya que son varias corrientes a lo interno de este movimiento) que "las filosofias de la liberación, convirtiéndose esta línea de filosofar en el fruto más representativo de la toma de conciencia de la identidad latinoamericana

4 En el 2003 -30 años después de los primeros encuentros y del primer manifiesto de la Filosofía de la Liberación- se reunieron en Rio Cuarto los fundadores de este movimiento. Cabe destacar que cada quien ha seguido su camino y es muy difícil encontrar una unidad en este heterogéneo movimiento.

5 Por ejemplo, a Horacio Cerutti-Gulberg por su trayectoria se le ha conferido el título de doctorado honoris causa en Polonia, y a Enrique Dussel en varias universidades, en reconocimiento del aporte de la Filosofía de la Liberación. En cuanto a la Teoria Crítica, sus teóricos y trayectoria (cf. Jay, 1989; Dubiel, 2000; Honneth, 2009). 
influencia de los frankfurtianos en lo que es el pensamiento crítico latinoamericano, fundamentalmente en la crítica a la "racionalidad dominante", a "la civilización burguesa occidental" y "el pensamiento triunfante" (Adorno y Horkheimer, 2013, pp. 11-17).

Lo cual es retomado, en particular, entre otras corrientes de pensamiento, por la Filosofía de la Liberación, añadiendo el horizonte de la liberación y el pensar dialéctico en relación con las transformaciones societales. Así lo concibió Adorno y Horkheimer (2013) en el prólogo a la nueva edición alemana de la Dialéctica de la Ilustración ${ }^{6}$ en 1969, cuando invocaron "al movimiento de la historia". Además decían:

Un pensamiento crítico que no se detenga ni ante el progreso exige hoy tomar partido por los residuos de libertad, por las tendencias al humanitarismo real, aunque parezcan impotentes frente a la potente marcha de la historia (Adorno y Horkheimer, 2013, p. 9).

Por otro lado, queremos ensayar la idea de Stefan Gandler (2011) de deslocalizar la Teoría Crítica, es decir, pensarla más allá de Frankfurt, más allá de Europa y Norteamérica, donde fueron sus focos de atención. En esa misma línea, analizar la idea de Enrique Dussel, de que la Filosofía de la Liberación es heredera de la Teoría Crítica $^{7}$. Y ello, dado que hay puntos de encuentro que retomar, en particular, por las nuevas generaciones.

A partir de estos, es imperante entablar un diálogo analógico entre Europa y Latinoamérica. Creo que aún se puede aprender de las experiencias ${ }^{8}$ de cada cual

6 Firmada por Adorno y Horkheimer en abril de 1969 en Franfurt am Main. Los autores decian: "de América, donde el libro se escribió, volvimos a Alemania con la convicción de poder hacer aqui más, en el plano teórico y en el práctico, que en ningún otro lugar". Esto es lo que Insausti (2015, pp. 110111) llama la "vuelta del exilio, vuelta a la Filosofía".

7 Enrique Dussel en un seminario en México en presencia de Albrecht Wellmer y Axel Honneth, y posteriormente en Berkeley con la presencia de Martin Jay y Judith Butler, pretendió demostrar que la herencia de las dos primeras generaciones de la Teoría Crítica es retomada por la Filosofía de la Liberación (cf. Dussel, 2015).

8 En Latinoamérica se han experimentado al menos estas dos últimas décadas, experiencias políticas enriquecedoras, que se plantearon otras formas de hacer las cosas; por un lado, los gobiernos posneoliberales (como Bolivia y Brasil por citar ejemplos); y por otra parte los movimientos antisistémicos (como el Ejercito Zapatista de Liberación Nacional y el Movimiento de los Trabajadores Rurales sin Tierra). 
en el terreno del pensamiento crítico. Esto es lo que consideró Nodal aquí, ya que la Filosofía de la Liberación retomó la crítica radical al estado actual de cosas, sin parangón, se fue a la raíz, a la crítica de la razón instrumental cuando muchos la daban como superada, o renegaban de ella. "Ya en 1970, Habermas [...] expresó su intención de no continuar la tradición de sus predecesores” (Insausti, 2015, p. 103).

Lo fundamental aquí, y es lo que queremos resaltar, es tener una actitud abierta al diálogo, de respeto primus inter pares, condición de posibilidad para el diálogo analógico de dos tradiciones de pensamiento de contextos distintos, pero que tienen la semejanza de criticar al sistema capitalista en el que viven. Otra cuestión no menos importante es que retoma el pensar teórico9.

Una de las cuestiones que no perdió el pensamiento crítico latinoamericano, y que en buena parte retoma la Filosofía de la Liberación, es lo utópico en el imaginario cultural, no en el sentido de lo ficticio, sino, en el de la transformación social ${ }^{10}$. Esto a pesar de la antiutopía del neoliberalismo -como diría Franz Hinkelammert (1984) - de que no exista utopía.

Al final, en el último epígrafe, analizaremos los puntos de entronque entre la Teoría Crítica y la Filosofía de la Liberación. Para que, a partir de ellos, trazar los puentes para mayor colaboración y producción de pensamiento crítico, en particular con las nuevas generaciones. Solo bajo este prisma podremos ir viendo la riqueza de la Teoría Crítica más allá de Frankfurt y los tiempos dorados de Adorno y Hokheimer, a los cuales sin duda hay que revisitar.

9 Ya en el siglo pasado Adorno y Horkheimer (2013, p. 13) anunciaban "la debilidad de la comprensión teórica". Esto tiene mucho que ver con las perspectivas y lecturas militantes. En muchas ocasiones los movimientos activistas ponen en un segundo plano la reflexión teórica, cuando deberian ir ambas cuestiones de la mano, la práctica y la teoría -al estilo de Hall (2010), de prácticas críticas -. Un ejemplo que lo ilustra es el de Ángela Davis, una probada y aguerrida activista por los derechos civiles, que no renegó de la reflexión teórica; asi estuvo cerca de Marcuse, fue estudiante de Adorno, y leyó a los clásicos del pensamiento crítico alemán: Kant, Hegel y Marx (cf. Davis, 2016). Esto lo señalo por cierto rechazo de los sectores militantes y de activistas a la reflexión teórica.

10 Para un análisis sistemático de lo utópico en nuestra América (cf. Ramírez, M., 2012). Que analiza la perspectiva utópica en Arturo Andres Roig, Horacio Cerutti-Guldberg, Fernando Ainsa y Franz Hinkelammert, entre otros. 


\section{Un primer momento}

La Teoría Crítica tiene sus raíces en la Escuela de Frankfurt y el Instituto de Investigación Social, a razón de que sus expositores fundadores se agruparon allí. Posteriormente la Teoría Crítica ira tomando su propia dinámica. El Instituto desde sus inicios fue autónomo, financiado con capital privado, vinculada a la Universidad de Frankfurt. En efecto, eso le permitió ser una institución formal con la libertad de estudiar abiertamente las contradicciones de la sociedad contemporánea.

Tiene particular importancia resaltar que se fundó en medio de guerras mundiales y vivió el totalitarismo nazi de forma directa. Los pensadores aglutinados en ese espacio, la mayoría eran judíos, como Theodor W. Adorno, Max Horkheimer, Leo Löwenthal, Erick Fromm, Herbert Marcuse, Walter Benjamin (los más conocidos) y muchos otros más, vivieron las consecuencias del antisemitismo en carne propia. Salieron de Alemania con diferentes destinos (con la excepción de Benjamin que no logró escapar $\left.{ }^{11}\right)$.

Pronto alcanzaron reconocimiento en Norteamérica y en varios países de Latinoamérica. Fue en medio de esta madeja de contradicciones que logran desarrollar propiamente lo que sería la Teoría Crítica. Es decir, que en momentos de grandes contradicciones surgen las grandes teorías.

En este sentido, tanto la Teoría Crítica y la Filosofía de la Liberación se tienen que comprender en movimiento con las transformaciones sociales en curso. Si nos percatamos de la evolución del pensamiento de los que fueron de la Escuela de Frankfurt, vemos una crítica a lo que están viviendo. Así, Fromm (1983, p. 8) criticó "las formas malignas del instinto destructor" del ser humano en sus numerosas variantes; Marcuse (2009), por otra parte, criticó -en este sentido, siguiendo a Adorno y Horkheimer sobre el pensamiento triunfante- "el triunfo del pensamiento positivo", que era "el pensamiento unidimensional ${ }^{12 "}$. Con esto quiero decir, que este tipo de pensamiento

11 Aún no está claro cómo fue la muerte de Benjamin. Algunos dicen que se suicidó, como señalan sus amigos Gershom Sholem y Hannah Arendt, otros se hacen eco del artículo de Stephen Schwartz: The mysterious death of Walter Benjamin en The Weekly Standard. Con la hipótesis de que a Benjamin lo mataron unos agentes rusos.

12 Que lo podiamos analizar como una farsa de la democracia occidental. Que tiene como telón de fondo la dominación y explotación de forma muy sutil que pasa desapercibida. 
era un pensamiento vivo, de carne y hueso ${ }^{13}$; no era una abstracción, y era heredero de la tradición teórica de la crítica de la sociedad. Todo esto que criticaron lo estamos viviendo en el siglo XXI de forma salvaje.

Cada tiempo tiene sus propias contradicciones, no es que hay que tomarse la Teoría Crítica tal cual la concibió Adorno y Horkheimer en el siglo XX; es necesario contextualizar la crítica, como diría Horkheimer (2013, p. 9) "no hay receta universal ${ }^{14 " . ~}$ Además de entender que la crítica tiene que ser sistemática. En este sentido, consideramos que la Filosofía de la Liberación es una contextualización de la Teoría Crítica ${ }^{15}$, en cuanto a crítica de la sociedad. Este es el entronque para un eventual diálogo de estas dos tradiciones.

Este diálogo es necesario y urgente, ya que al parecer la Teoría Crítica necesita actualizarse, seguir más adelante de donde Habermas, Apel y Honneth llegaron y están. Esto lo vemos en la "necesidad de repensar el concepto de crítica frente al modelo de J. Habermas y A. Honneth” (Romero, 2016); “¿qué hay de crítico en la Teoría Crítica? El caso de Habermas y el género" (Fraser, 2015); y una vuelta a “Adorno y Hegel” (Insausti, 2015). Esto deja entrever la necesidad de repensar y abrirse hacia nuevas condiciones de posibilidad de una Teoría Crítica que esté al tanto críticamente de los problemas del siglo XXI.

\section{Recepción}

Para ver la recepción de las críticas que se fraguaron desde la denominada Escuela de Frankfurt, que luego serían el boom de la Teoría Crítica en Latinoamérica, hay que

13 Como diria Marx en los Grundrisse, en el método se requiere empezar por lo "real y concreto" para así desplegarse hacia lo general, llegado alli, emprender un viaje de retorno, asi se tendria un conocimiento de las múltiples determinaciones, que sería lo concreto, "la unidad de lo diverso" (cf. El método de la economía política, [§ 22], Marx, 1971, p. 21).

14 Esto tiene gran importancia para el eventual diálogo entre las nuevas tomas de posición de la Teoría Crítica y la Filosofía de la Liberación, ya que uno de los principales problemas de vieja data para una relación analógica, condición sine qua non para un diálogo real, es romper con la pretensión de universalidad que tienen en su interior el pensamiento eurocéntrico, como ideología -diría Samir Amin (1989)-, y que muchos de los pensadores europeos y norteamericanos mantienen.

15 No únicamente, también hay otros esfuerzos interesantes. Como el de Veraza (2014) de leer a Sade en clave política, recodando un poco el Excurso II de la Dialéctica de la llustración de Adorno y Horkheimer (2013). Esto demuestra lo rico del pensamiento crítico latinoamericano que no renuncia a la tradición teórica y la crítica sistemática de la modernidad, donde Sade es una figura enigmática. 
ver los vínculos que se dieron a mediados del siglo pasado, lo que se hace evidente materialmente en la publicación de artículos, libros, reseñas, estudios introductorios, prólogos, ediciones y traducciones de estos autores al castellano, tomando en cuenta el sentido de las traducciones y las lecturas que se hacen (que varían según el interés). Estos elementos nos permiten analizar el alcance y los límites de la influencia, ya que fue asimétrica.

Por eso, nos tenemos que retrotraer a ese primer momento donde muchos miembros de la Escuela de Frankfurt emigraron, algunos llegaron a Latinoamérica, entablando así los primeros vínculos, otros fueron a Norteamérica donde se había afiliado el Instituto en la Universidad de Columbia, gracias a Nicholas Murray Butler ${ }^{16}$, donde llegaron sus figuras canonícas, como Marcuse, Lowenthal, Adorno y Horkheimer.

Para concretizar, es necesario centrarse en algunos aspectos, por ejemplo: la publicación en castellano de estos autores, la presencia física de estos, que emigraron mayormente a partir del ascenso del nazismo. Como señalamos, la lectura que hacen los latinoamericanos de la Teoría Crítica, es fundamental para saber qué extrajeron, interpretaron y aplicaron a los núcleos problematizadores que surgían de la necesidad de pensar desde la realidad latinoamericana.

El primer aspecto, el de las publicaciones, tiene varios momentos. Estas se dieron en diferentes espacios geográficos, como en México, Centro y Sur América. En Panamá, ya a finales de la década del treinta, había algunos artículos traducidos. En Buenos Aires, desde la revista Sur, a finales de la década del sesenta, y en Caracas en la editorial Monte Ávila se traducían otros tantos.

En Panamá, algunos profesores alemanes directamente vinculados a la Escuela de Frankfurt dictaron clases, como Borkenau que enseñaba en Londres y Honigsheim en Paris, publicaron artículos y fundaron institutos de investigación. En general las traducciones del alemán al castellano en Latinoamérica fueron muy fructíferas. En el sur fueron acogidas las críticas de la Teoría Crítica.

Vamos a ver algunos vínculos y relaciones que se dieron de los pensadores que se asociaban con la Escuela de Frankfurt en países latinoamericanos (como Panamá,

16 Fue rector de la Universidad de Columbia, Premio Nobel de la Paz en 1931, y quien le abrió las puertas a Horkheimer para que el Instituto tuviese una sede en Estados Unidos. 
México y Argentina ${ }^{17}$ ). A partir de la segunda mitad del siglo XX, ya podemos hablar propiamente de una recepción de la Teoría Crítica, que tomará su propio camino. Luego de pasar revisión a estos vínculos, volveremos sobre la cuestión de la necesidad del diálogo analógico de la Teoría Crítica y la Filosofía de la Liberación.

\subsection{En Argentina}

Uno de los primeros vínculos de la Escuela de Frankfurt con Argentina se remonta a los primeros días de su fundación. Fue con el financiamiento de los Weil (particularmente por la insistencia de Félix Weil, nacido en Argentina) que tenían una gran fortuna gracias a la exportación de granos, que se fundó el Instituto a principios del siglo XX, esto es lo que se conocería popularmente como Escuela de Frankfurt, la cual se convertiría en uno de los mayores centros de producción y difusión de pensamiento crítico mundial.

Otro vínculo es la traducción de algunos de los trabajos centrales de la TC. Por ejemplo, la revista (y editorial) Sur dirigida por Victoria Ocampo, quien realizara la edición y traducción de la colección Estudios alemanes ${ }^{18}$, entre 1966 y 1970. Esta revista (y editorial) no era afín necesariamente a la Teoría Crítica. Esta colección fue de autores alemanes en general, pero coincidió que se tradujo y publicó los trabajos centrales de la Teoría Crítica. Pese a los conflictos de qué motivó estas traducciones, lo cierto es que sirvió de plataforma (tomando en cuenta las limitaciones idiomáticas) para difundir lo que sería la Teoría Crítica en Argentina y el resto de Latinoamérica.

\subsection{En Panamá}

A partir de 1935 con la fundación de la Universidad de Panamá (UP), varios profesores alemanes vinculados a la Escuela de Frankfurt, llenaron e innovaron las cátedras nacionales y contribuyeron al desarrollo de las Humanidades y las Ciencias

17 Esto no quiere decir que solo se dieron en estos tres países, pero con estos podremos tener al menos una idea concreta de la relación.

18 Dentro de esta colección estaban los siguientes trabajos, entre paréntesis las fechas en que se publicaron: Filosofía de la nueva música de Theodor W. Adorno (1966), Teoría y praxis de Jürgen Habermas (1966), Ensayos escogidos de Walter Benjamin (1967), Cultura y sociedad de Herbert Marcuse (1967), Crítica de la razón instrumental de Max Horkheimer (1969), Dialéctica del iluminismo de Adorno y Horkheimer (1970) y Sobre el concepto de hombre y otros ensayos de Horkheimer (1970). 
Sociales en la Universidad de Panamá19. Por ejemplo Paul Honigsheim, "cabeza de la filial del Instituto de Investigación Social en París" (Jay, 1989, p. 78), dictó todos los cursos de Filosofia, teniendo como estudiante a Diego Domínguez Caballero. Franz Borkenau dictó la cátedra de Civilización e Historia y publicó, como también lo hiciera Honigsheim, algunos artículos en 1936 en la revista Universidad de Panamá.

En términos generales, muchos alemanes fortalecieron el desarrollo de las Humanidades y las Ciencias Sociales en Latinoamérica. Eso ocurrió en Panamá, fortalecieron a la naciente academia panameña y a la Universidad de Panamá en particular, a tal punto que su rector Octavio Méndez Pereira les solicitó quedarse, pero este no era el horizonte de estos profesores que luego regresarían a Alemania o harían carrera en Norteamérica u otros países del sur.

\subsection{En México}

Desde la década del cuarenta ya hay traducciones de miembros de la Escuela de Frankfurt en México, para 1943 aparece el Fondo de Cultura Económica Behemont. Pensamiento y acción en el Nacional Socialismo de Franz Leopold Neumann. Dos años más tarde el recién creado Colegio de México edita En busca de la soberanía de Otto Kirchheimer.

Erich Fromm gozó de muy buena recepción en la Universidad Nacional Autónoma de México (UNAM), donde fueron editadas sus obras, incluso colaboró con la editorial Fondo de Cultura Económica. Vivió más de dos décadas en México, su primera visita (en 1935) fue muy fructífera y pronto estableció el puente entre la UNAM y pensadores como Herbert Marcuse, este dictó una serie de conferencias en enero de 1966 en la Escuela de Ciencias Políticas, las cuales abrieron el debate en torno a la Teoría Crítica.

En la década del sesenta la UNAM se convirtió en un foco para los debates más acuciantes de las contradicciones de la sociedad contemporánea, cuestión donde los alemanes eran pioneros, dadas las circunstancias que habían vivido bajo el totalitarismo nazi.

19 Martin Jay (1989) en su historia de la Escuela de Frankfurt escribe sobre Paul Honigsheim y Franz Borkenau, sus primeros años y participación en el Instituto de Investigación Social, que fueron figuras prestantes. Sobre el aporte de los alemanes en las Ciencias Sociales y las Humanidades en la Universidad de Panamá, (cf. Figueroa, 1998; Porcell 1991). 
Fromm, que ya tenía una presencia bien marcada en México, influyó en la incorporación del psicoanálisis a los estudios de medicina clínica. Dictó varias conferencias y constituyó varias instituciones, por ejemplo: la Sociedad Psicoanalítica Mexicana y el Instituto Mexicano de Psicoanálisis. Participó en revistas y ediciones de libros, el aporte de Fromm se orientó particularmente en introducir la noción de "psicoanálisis humanista" en las humanidades, las ciencias sociales y la medicina, no dudamos la incidencia que adquirió en toda la académica mexicana este aporte.

Walter Benjamin también influyó en México, actualmente es muy discutido, la reciente compilación La mirada del ángel. En torno a las tesis sobre la historia de Walter Benjamin realizada por Bolívar Echeverría (2005) da muestra de ello. Además que lo podemos considerar un clásico del pensamiento crítico, que nunca termina de decir lo que tiene que decir.

Las ideas de Benjamin son recontextualizadas en la academia mexicana, por ejemplo, la última frase de la VII Tesis de filosofía de la historia, "pasarle a la historia el cepillo a contrapelo" (Benjamin, 1989, p. 182) es concebida frecuentemente como una crítica a la historia oficial muy dominante en las mentalidades, en ese sentido, la noción de contrahistoria que implementa, por ejemplo, Carlos Aguirre Rojas ${ }^{20}$ para analizar la historia de México, mucho se asemeja al contrapelo benjaminiano.

También retomando la idea de Stefan Gandler, que resulta interesante, de pensar la Teoría Crítica sin Frankfurt que señala que "en México se podría ver como un muy buen candidato a Bolívar Echeverría, quien, desde nuestra perspectiva, aporta más a algo como una nueva Teoría Crítica" (Gandler, 2011, p. 114). Bolivar Echeverría ${ }^{21}$ murió en el 2010, relativamente joven, con 69 años, pero dejó muchos discípulos, como el mismo Gandler, que seguirán aportando sustantivamente a la crítica de la modernidad, como en efecto lo hacía el maestro Echeverría.

El despliegue hacia otras latitudes de la Teoría Crítica se debe en gran medida a que los autores de las posteriores generaciones -a la primera- de la Escuela de Frankfurt renunciaron (con excepciones) al pensamiento crítico $^{22}$, o se han resignado al status quo.

20 Su trabajo se puede consultar en: http://www.contrahistorias.com.mx/

21 Su trabajo se puede consultar en: http://www.bolivare.unam.mx/

22 Podriamos decir que a un pensamiento conciliador en unos casos y conformista en otros. El caso más emblemático es el de Jürgen Habermas. Pensar el legado de la Teoría Crítica sin sus pensadores actuales 


\section{Un segundo momento. Retomar la radicalidad}

Después de pasar revisión de algunos hechos concretos de la relación de la Escuela de Frankfurt con Latinoamérica. Ahora podemos ir aterrizando en lo que corresponde propiamente a las cuestiones para el diálogo analógico ${ }^{23}$ de la Teoría Crítica y la Filosofía de la Liberación; que es posible a partir de los puntos de entronque. Por diálogo analógico entendemos aquel en el que se respeta a los participantes a pesar de que los planteamientos sean distintos.

Retomar la radicalidad ${ }^{24}$ con que fue expuesta la crítica de la Teoría Crítica, es la tarea pendiente. Fundamentalmente de las nuevas generaciones, que deben retomar las expresiones más críticas de la tradición y abrir sus propios caminos. La historia sigue su curso.

Para contextualizar un poco, en Latinoamérica era necesario un pensamiento críti$\mathrm{CO}^{25}$ que no renunciara a su historia y tradición, pero que no se cerrara en sí mismo. La crítica al totalitarismo, a la sociedad capitalista y a la ortodoxia marxista de los frankfurtianos ayudó a complementarlo.

se debe a ese viraje que han dado con respecto a esa primera generación (hay que tomar en cuenta que el mundo cambió, y no son los mismos problemas que tiene la sociedad). Para la crítica a este viraje de Helmut Dubiel, Axel Honneth y Jürgen Habermas, véase: Gandler (2011). Sobre Habermas también habría que añadir objetivamente un cambio sustancial del "desplazamiento del paradigma de la filosofía de la conciencia al que estaba vinculada la Teoría Crítica de la sociedad [....] al paradigma de la acción comunicativa" (Aguirre, 1991, p. 415). Para José María Aguirre (1991) no es una ruptura, sino un continuum reactualizado de la Teoria Crítica, pero creemos oportuna tal distinción entre un primer Habermas de los años setenta con un segundo Habermas, el de la Teoría de la acción comunicativa en 1981.

23 En esto insistió Dussel en el curso: "Sobre el método analéctico crítico", en el semestre 2016-2, en el Colegio de Filosofía de la UNAM.

24 Como ya hemos dicho, no es exclusividad de la Filosofía de la Liberación retomar la radicalidad de la Teoría Crítica, hay otros intentos interesantes, ya citamos arriba el de Veraza. Otro ejemplo es el de la "Filosofia Radical" de Frieder Otto Wolf. Su trabajo se puede consultar en: http://www.friederottowolf.de

25 Recientemente el Concejo Latinoamericano de Ciencias Sociales (CLACSO) editó la colección Antologías del pensamiento social latinoamericano y caribeño. Que reúne varias antologías por paises, de lo más destacado del Pensamiento Crítico contemporáneo. Además, de otra, de los autores coralarios del siglo pasado, se coeditó con Siglo XXI Editores. Entre los que están: Ruy Mauro Marini, Carmen Miró, Edelberto Torres-Rivas, Pablo González Casanova, Agustín Cueva, Enzo Faletto, René Zavaleta, Florestan Fernandes, Orlando Fals Borda y Álvaro Garcia Linera. 
Retomando la idea de Enrique Dussel de que la Filosofía de la Liberación es heredera de la Teoría Crítica, en cuanto a crítica sistemática ${ }^{26}$. Esto no quiere decir que la Filosofía de la Liberación se deba o sea el resultado de la Teoría Crítica, cronológicamente podría ser, pero contextualmente nace de otro núcleo de problemas, sería muy forzado decir que parten de los mismos problemas, e irreflexivo negarlo. Comparten la crítica al sistema capitalista, y sus formas de expresión según el contexto, ya que no es lo mismo el desarrollo del capitalismo en Inglaterra que en Panamá, por ejemplo.

Para ver los detalles de cada caso, y las semejanzas, es necesario un diálogo analógico entre estas dos tradiciones, para ajustar cuentas y abrir nuevos espacios. Así, conocer de primera mano las perspectivas, que no necesariamente tengan la misma lógica discursiva, la misma dinámica, sino que aportan mayor diversidad, mayor unidad a partir de lo diverso ${ }^{27}$. Lo cual sería enriquecedor para el pensamiento crítico mundial.

En Latinoamérica, surgió la Teoría de la Dependencia y la Teología de la Liberación, también estaba en boga la disputa de Augusto Salazar Bondy y Leopoldo Zea (19681969), este primero apelaba a la dificultad de una filosofía autentica -en el contexto latinoamericano- por estar inmersa en una cultura de dominación, en cambio Zea esforzándose sin más por pensar la cultura y la historia latinoamericana, para a partir de allí, hacer una filosofía latinoamericana. Diríamos que estos son los antecedentes mínimos de la Filosofía de la Liberación ${ }^{28}$.

En México y en Berkeley, Enrique Dussel presentó la idea de que la herencia de las dos primeras generaciones de la Teoría Crítica de Frankfurt es la Filosofía de la Liberación, en presencia de Axel Honneth (en la UAM-I) y luego de Martin Jay y Judith Butler (en la Universidad de California), entre otros. Es decir, esta idea no fue una provocación sin resonancias, sino que se presentó a lo interno del mismo debate sobre la actualidad de la Teoría Crítica, en presencia de sus teóricos más importantes.

26 A esto responde la Política de la liberación (está previsto que sea una trilogía, de lo cual han salido dos tomos publicados por Trotta) de Enrique Dussel.

27 Esta idea la trabajó ampliamente Hegel y Marx, y en Latinoamérica Camilo Torres Restrepo.

28 Para una visión sistemática de la Filosofía de la Liberación (cf. Cerutti-Guldberg, 2006) que añadió una nota amplia de referencias bibliográficas actualizadas a la fecha. Además (cf. Scannone J. C. 2009). 
Esta herencia, de la que habla Dussel, no se trata de la implementación de los supuestos de la Teoría Crítica en el contexto latinoamericano ${ }^{29}$. La Filosofía de Liberación es una propuesta que se piensa críticamente desde el lugar que se enuncia, es crítica de lo convencional, lo conformista, lo reformista o lo que Adorno y Horkheimer (2013) llaman el pensamiento triunfante. Siempre se está renovando al calor de las propias contradicciones que se viven en Latinoamérica ${ }^{30}$. Una región que cuenta con grandes riquezas, pero vive grandes desigualdades.

Dada esas circunstancias, hay que retomar el tema de "la materialidad (corporal, ecológica, económica, cultural)" (Dussel, 2015, p. 17), de la victima del sistema capitalista $^{31}$. Esto es lo que Hinkelammert (2013) llama una fenomenologia de la vida real, que consiste en "la realidad vivida". Por ello, hay que poner al ser humano como principio material de toda posibilidad de crítica al sistema, como fundamento último. Se podría interpretar como una vuelta al materialismo ${ }^{32}$ que tenga como norte la vida real $^{33}$.

La Filosofía de Liberación se presenta al siglo XXI como lo fue desde su origen: crítica y diversa, pero que en su núcleo problemático -como lo han expresado en el Manifiesto de Rio Cuarto ${ }^{34}$ - contiene los temas más acuciantes de nuestro siglo; el rechazo al proceso de la globalización neoliberal, la especulación financiera, el desempleo

29 Tampoco se puede negar la influencia que "se produce a través de la llamada primera generación de la Escuela de Frankfurt, sobre todo M. Horkheimer, Th. Adorno y H. Marcusse" (Beorlegui, 2010, p. 665). Sobre esto, también habla Cerutti-Guldberg (2006) ampliamente.

30 A esto responde el último libro de Cerutti-Guldberg (2015): Posibilitar otra vida transcapitalista. Que integra la problemática ética, epistémica y política que vive la región en un contexto global.

31 Aunado a esto, está la crítica a la conciencia moderna que sería en todo caso la crítica inmanente al capitalismo. Por eso, Enrique Dussel estará dando el curso Dialéctica de la modernidad: origen, desarrollo y ocaso. En el semestre 2017-1, en el Colegio de Filosofía - UNAM. También esta crítica está presente en autores como Juan José Bautista, y los teóricos del Giro Decolonial como Ramón Grosfoguel, Walter Mignolo, Nelson Maldonado-Torres, Maria Lugones, Catherine Walsh, Yuderkis Espinosa y muchos otros.

32 La vuelta al materialismo se ha expresado de diversas formas. Por ejemplo, siempre es una constante en los trabajos de Perry Anderson; y en los últimos años Slavoj Zizek y Alain Badiou tienen un proyecto de una vuelta al materialismo dialéctico. Por otra parte también está el proyecto Cosmos de Michel Onfray.

33 Para esta perspectiva (cf. Hinkelammert, 2013), en particular el § 10, Sobre la reconstitución del pensamiento crítico.

34 Firmado con ocasión de la convocatoria efectuada por el Intercambio Cultural Alemán-Latinoamericano (ICALA) de Rio Cuarto, Argentina, a los 7 dias del mes de noviembre de 2003. Por: Alberto Parisi, Aníbal 
estructural, la brecha entre pobres y ricos, la exclusión en todas sus formas, la uniformidad cultural, cuestiones que afectan la dignidad humana y la libre determinación de los pueblos. En fin, cuestiones que expresan una preocupación que solo puede ser redimida desde el diálogo analógico entre las diversas perspectivas críticas.

\section{Referencias}

Adorno, T., y Horkheimer, M. (2013). Dialéctica de la ilustración. Fragmentos filosóficos. Madrid: Akal.

Aguirre, O. (1991). Habermas: teoría del conocimiento y teoría de la sociedad. Pensamiento. Revista de investigación e información filosófica, 47(188), 415-448.

Amin, S. (1989). El eurocentrismo. Crítica de una ideología. 1. a ed. México: Siglo XXI.

Benjamin, W. (1989). Discursos interrumpidos I. Filosofía del arte y de la historia. Argentina: Taurus.

Beorlegui, C. (2010). Historia del pensamiento filosófico latinoamericano. Una búsqueda incesante de la identidad. 3. ${ }^{\text {e }}$. Bilbao: Universidad de Deusto.

Cerutti-Guldberg, H. (2006). Filosofía de la liberación latinoamericana. 3. a ed. México: Fondo de Cultura Económica.

Cerutti-Guldber, H. (2015). Posibilitar otra vida trans-capitalista. 1. ${ }^{a}$ ed. México: UNAM / CIALC.

Davis, A. (2016). Autobiografía. Madrid: Capitan Swing.

Del Rayo Ramírez, M. (2012). Utopología desde nuestra América. 1. a ed. Bogotá: Ediciones desde abajo.

Dubiel, H. (2000). La teoría crítica. 1. a ed. México: UAM-I, DAAD, Instituo Goethe, Plaza y Valdés.

Dussel, E. (2011). Filosofía de la Liberación. México: Fondo de Cultura Económica.

Fornari, Enrique Dussel, Horacio Cerutti, Juan Carlos Scanonne, Antonio Kinen, Mario Casalla, Carlos Cullen, Julio de Zan y Arturo Andrés Roig. Se puede consultar en: http://old.afyl.org/manifiesto.html 
Dussel, E. (2015). Filosofías del sur. Descolonización y transmodernidad. México: Akal.

Dussel, E. (2016). 14 tesis de ética. Hacia la esencia del pensamiento crítico. 1. ${ }^{\mathrm{a}}$ ed. Madrid: Trotta.

Echeverría, B. (Comp.). (2005). La mirada del ángel. En torno a las tesis sobre la historia de Walter Benjamin. México: ERA-UNAM.

Figueroa, A. (1998). Las Ciencias Sociales en Panamá en víspera del tercer milenio. Panamá: Editorial Portobelo.

Fraser, N. (2015). Fortunas del feminismo. 1. a ed. Madrid: Traficantes de sueños.

Fromm, E. (1983). El corazón del hombre. 1. ${ }^{\mathrm{a}}$ ed. México: Fondo de Cultura Económica.

Gandler, S. (2000). Marxismo crítico en México: Adolfo Sánchez Vázquez y Bolívar Echeverría. México: FCE-UNAM-Universidad Autónoma de Querétaro.

Gandler, S. (2011). Fragmentos de Frankfurt. Ensayos sobre la teoría crítica. México: Universidad Autónoma de Querétaro.

Habermas, J. (1993). El discurso filosófico de la modernidad. Madrid: Taurus.

Hall, S. (2010). Sin garantías: trayectorias y problemáticas en estudios culturales. 1. ${ }^{\mathrm{a}} \mathrm{ed}$. Colombia, Lima, Quito: Envión editores, Instituto de Estudios Peruanos, Instituto de Estudios Sociales y Culturales, Pensar. Universidad Javeriana, Universidad Andina Simón Bolívar, sede Ecuador.

Hinkelammert, F. (1984). Crítica a la razón utópica. 1. a ed. San José: DEI.

Hinkelammert, F. (2013). La maldición que pesa sobre la ley. Las raíces del pensamiento crítico en Pablo de Tarso. 2. ${ }^{\mathrm{a}}$ ed. San José: Arlekín.

Honneth, A. (2009). Patologías de la razón. Historia y actualidad de la teoría crítica. Buenos Aires: Katz.

Horkheimer, M. (2003). Teoría crítica. 1. ${ }^{\mathrm{a}}$ ed. Buenos Aires-Madrid: Amorrortu.

Insausti, X. (2015). Adorno y Hegel: avatares de una larga y compleja ralación. En Aurrekoetxea, A., y Fernando, G. (Eds.). Prismas críticos. Lecturas sobre Theodor W. Adorno (pp. 103-122). Granada: Comares. 
Insausti, X. (2015). Ideas para una filosofía crítica en el siglo XXI. En Insausti, X., Nogueroles, M., y Vergara, J. (Eds). Nuevos diálogos de pensamiento crítico (pp. 119-131). Madrid: UAM, UPV, RIPC.

Jay, M. (1989). La imaginación dialéctica. Una historia de la Escuela de Frankfurt. Madrid: Taurus.

Marcuse, H. (2009). El hombre unidimensional. Ensayo sobre la ideología de la sociedad industrial avanzada. 9. ${ }^{\text {e }}$. Barcelona: Ariel.

Marx, K., y Engels, F. (1976). El manifiesto comunista. 3. a ed. Madrid: Ayuso.

Marx, K. (1971). Elemento fundamentales para la crítica de la economía política. Grundrisse. (Borrador), 1857-1858. 1. ${ }^{\text {a }}$ ed. Madrid: Siglo XXI.

Porcell, N. (1991). Los docentes europeos y la formación de la Universidad de Panamá. Panamá: Universidad de Panamá-ICASE.

Romero, J. (2016). El lugar de la crítica. Teoria crítica, hermeneutica y el problema de la trascendencia intrahistórica. 1. a ed. Madrid: Biblioteca Nueva.

Scannone, J. C. (2009). La filosofía de la liberación: historia, características, vigencia actual. Teología y vida, 50, 59-73.

Veraza, J. (2014). El otro Sade. Democracia directa y crítica integral de la modernidad. 1. ${ }^{a}$ ed. México: Itaca. 